\title{
Beauty and Charm Physics at CDF Run II
}

\author{
M. Bishai ${ }^{1}$ (for the CDF II collaboration) \\ Fermilab National Laboratory, P.O. Box 500 \\ Batavia, IL 60510-0500, U.S.A \\ e-mail: bishai@fnal.gov
}

Received:

\begin{abstract}
Using the data samples collected with the CDF Run II detector during the year 2002 and early 2003, new measurements of the production cross-sections and the masses, lifetimes and branching fractions of beauty and charm hadrons are presented. New measurements of the $\Lambda_{b}$ mass, lifetime, and branching fractions have greatly improved the current knowledge of bottom baryon properties and decay dynamics. The large charm signals made available by the silicon vertex track trigger have enabled the establishment of key measurements using rare charm decays that are sensitive to new physics beyond the Standard Model. The decay signals $B_{s} \rightarrow D_{s} \pi$ and the two body charmless decays of $B^{0}$ and $B_{s}$ have been established. These decay channels are important milestones towards the measurement of $B_{s}$ mixing and direct $\mathrm{CP}$ violation in the $B$ system.
\end{abstract}

PACS: $13.85 . \mathrm{Ni} ; 13.85 . \mathrm{Lg} ; 13.25 . \mathrm{Hw} ; 12.38 . \mathrm{Qk} ; 13.30 . \mathrm{Eg} ; 12.15 . \mathrm{Ff} ; 12.15 . \mathrm{Mm}$

\section{Introduction}

The CDF Run II detector is described in detail in reference [1]. In CDF II, the Silicon Vertex Tracker (SVT) [2] reconstructs two dimensional tracks in 4-5 layers of the inner silicon vertex detector online, thus allowing triggers on detached vertices at Level 2. The SVT impact parameter resolution is measured to be $48 \mu \mathrm{m}$ including a $30 \mu \mathrm{m}$ beam spot. The SVT selects tracks with transverse momenta greater than $2 \mathrm{GeV} / \mathrm{c}$ and impact parameter to the beam $>120 \mu \mathrm{m}$. The SVT reconstruction efficiency versus track transverse momentum and impact parameter is shown in Figure 1. Since January 2002, large samples of $D$ and $B$ mesons from $B \rightarrow D X$ decays have been collected using SVT. Approximately 1 million fully reconstructed charm decays have been collected in the first 138 $\mathrm{pb}^{-1}$ of data.

The Run II Level 1 muon triggers have also been upgraded. The muon triggers combine tracks reconstructed in the central-outer tracker drift chamber (COT) with muon track stubs in the central muon systems. The triggered muon transverse momentum threshold is at $1.5 \mathrm{GeV} / \mathrm{c}$, where the Level 1 muon trigger efficiency is $92 \%$. The muon trigger efficiency is $97 \%$ for muons with transverse momentum $>2 \mathrm{GeV} / \mathrm{c}$. A large sample of $J / \psi$ events has been collected using the di-muon triggers (more than 1 million $J / \psi$ in $138 \mathrm{pb}^{-1}$.) 

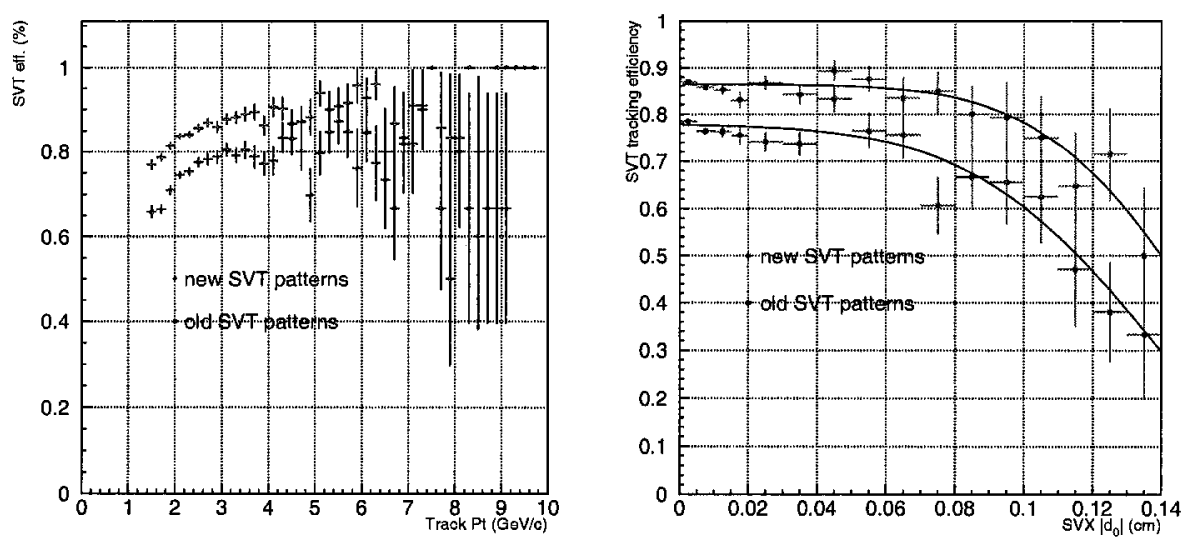

Fig. 1. SVT efficiency compared to offline reconstruction as a function of track transverse momentum (left) and impact parameter (right). The distributions labeled new indicate the improvement in SVT performance achieved in mid2003.

In this paper, the CDF Run II beauty and charm measurements of production cross-sections, masses, lifetimes, and rare decays from data collected during 2002 and early 2003, with the upgraded SVT and di-muon triggers, are reviewed.

\section{Charm Production}

\subsection{Direct Charm Production Cross-section}

The secondary vertex trigger immediately provided large samples of fully reconstructed $D$ mesons during the first few months of data taking in early 2002 . From $5.7 \mathrm{pb}^{-1}$ of data, $D$ mesons were reconstructed in the modes $D^{0} \rightarrow K^{-} \pi^{+}$, $D^{*+} \rightarrow D^{0} \pi^{+}, D^{0} \rightarrow K^{-} \pi^{+}, D^{+} \rightarrow K^{-} \pi^{+} \pi^{+}$, and $D_{s} \rightarrow \phi \pi^{+}$as shown in Figure 2. Charm mesons originating from $B$ meson decays can be distinguished from directly produced charm by studying the impact parameter of the meson to the primary vertex. $D$ mesons from secondary decays of $B$ mesons will not point back to the primary vertex. A Monte Carlo simulation (MC) is used to model the impact parameter distribution of charm mesons from $B$ decays. The impact parameter distribution of two-track vertices that originate from the primary vertex is measured using $K_{s} \rightarrow \pi \pi$ decays in the same data sample. The fit to the impact parameter distribution of reconstructed $D$ mesons is shown in Figure 3. The fraction of directly produced $D$ mesons obtained from the fit is $f_{D}\left(D^{0}\right)=86.6 \pm 0.4 \pm 3.5 \%, f_{D}\left(D^{*+}\right)=88.1 \pm 0.4 \pm 3.5 \%$, $f_{D}\left(D^{+}\right)=89.1 \pm 0.4 \pm 2.8 \%$ and $f_{D}\left(D_{s}^{+}\right)=77.3 \pm 3.8 \pm 2.1 \%$. The number of observed directly produced $D$ mesons is corrected for the tracking and secondary vertex trigger efficiencies and detector acceptance. The differential cross-section thus obtained, for various $D$ mesons with transverse momentum $p_{T}>6.0 \mathrm{GeV} / \mathrm{c}$, 
and rapidity, $|y|<1.0$, is shown in Figure 4 with the theoretical prediction from recent NLO QCD calculations [3] overlaid. The data is found to be $30-70 \%$ higher than current theoretical predictions.
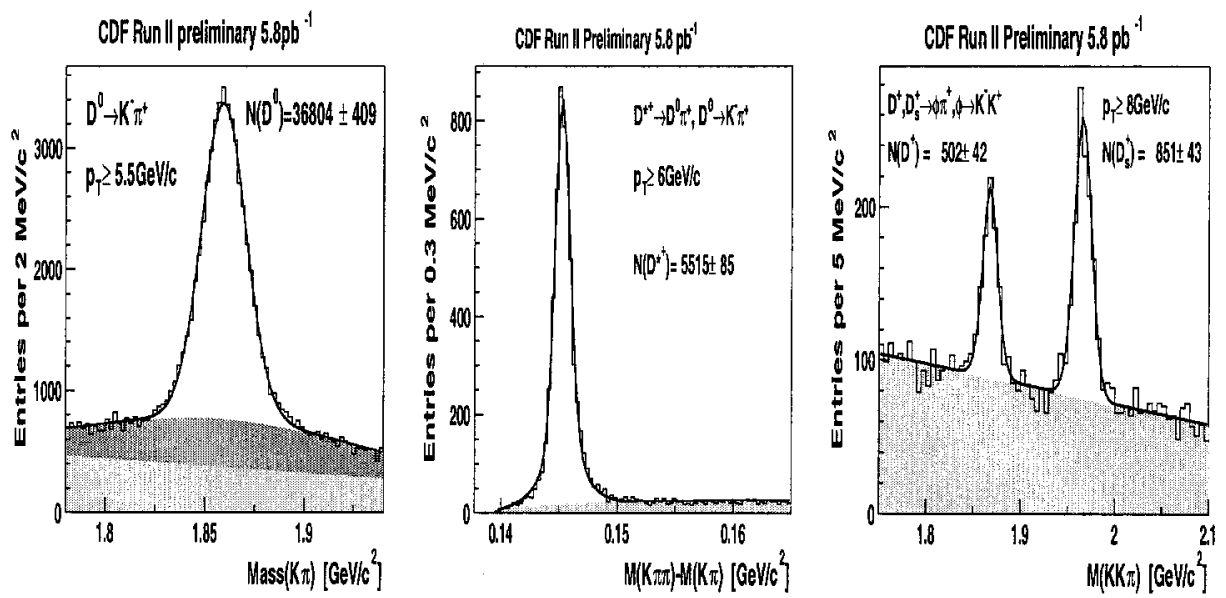

Fig. 2. $D^{0}, D^{*}$, and $D_{s}$ invariant mass signals reconstructed in CDF Run II
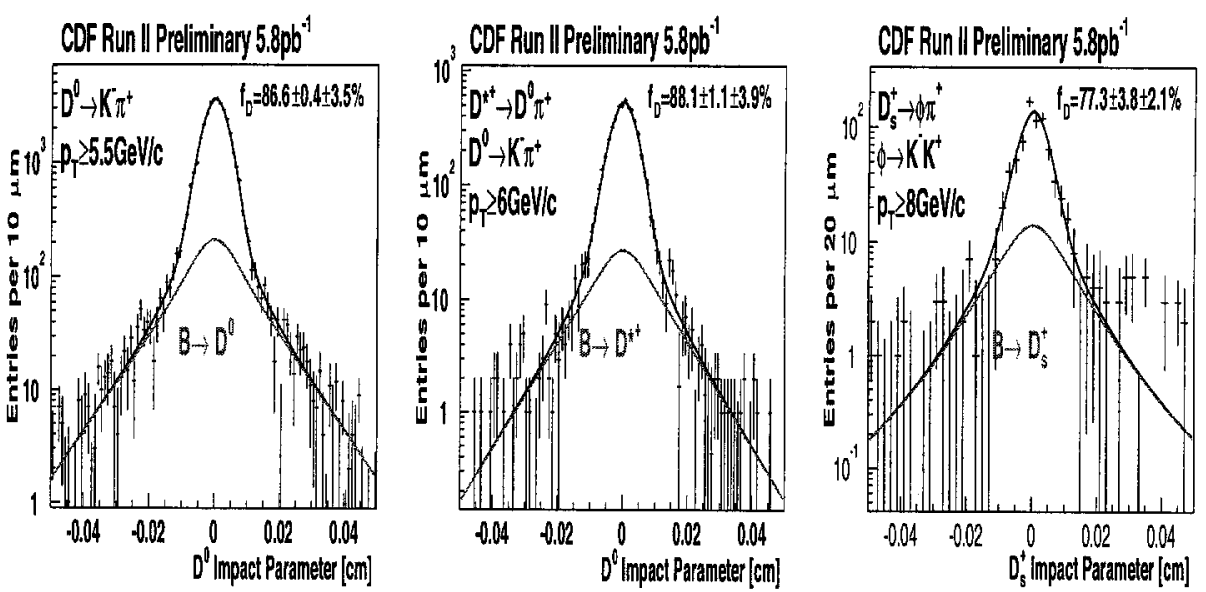

Fig. 3. Binned likelihood fit to the sideband subtracted $D$ impact parameter distributions used to determine the fraction of prompt $D$ mesons in the inclusive modes.

\subsection{Charmonium Production}

Non-relativistic quarkonia bound states are best described by Non-Relativistic QCD ( NRQCD) theoretical models which are used to predict the hadroproduction cross-sections [4]. At large transverse momenta, fragmentation type 

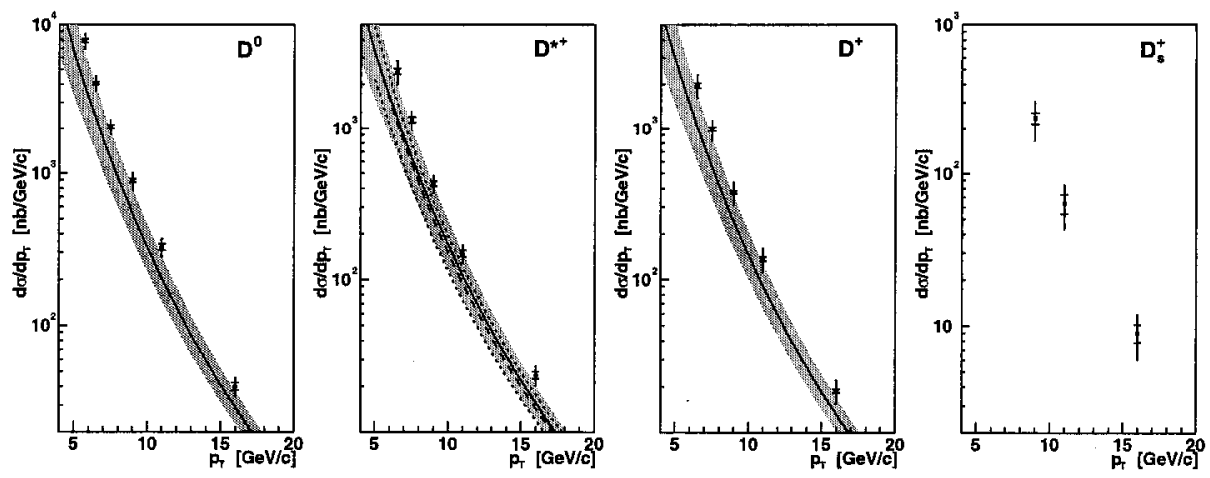

Fig. 4. The differential $D^{0}, D^{*}, D^{+}$, and $D_{s}$ cross-section distributions measured in CDF Run II ( points with error bars) and the theoretical predictions overlaid as a shaded band.

production is expected to dominate and color-octet matrix elements dominate the color-singlet matrix element contribution. Using color-octet matrix elements extracted from data, the model can accommodate the Run I data at the Tevatron for $J / \psi$ with transverse momenta $>5 \mathrm{GeV} / \mathrm{c}$. At low transverse momenta, soft gluon effects and non-fragmentation effects from other octet matrix elements that are difficult to calculate theoretically become important and cause theory predictions and data to diverge. The Run-II CDF detector has an improved dimuon trigger with a lower muon transverse momentum threshold of 1.5 $\mathrm{GeV} / \mathrm{c}$. This has extended the low transverse momentum range of triggered $J / \psi$ events down to $p_{T}=0 \mathrm{GeV} / \mathrm{c}$. A new measurement of the total inclusive $J / \psi$ cross-section in the rapidity range $|y|<0.6$ using $37 \mathrm{pb}^{-1}$ of Run-II data has been carried out. This is the first measurement at a hadron collider of the total cross-section of centrally produced $J / \psi$. The differential cross-section is shown in Figure 5 and the integrated cross-section is measured to be:

$$
\sigma(p \bar{p} \rightarrow J / \psi X,|y(J / \psi)|<0.6) \times B r(J / \psi \rightarrow \mu \mu)=240 \pm 1(\text { stat })_{-28}^{+35}(\text { syst }) \mathrm{nb}
$$

\section{Masses, lifetimes, and Branching Fractions of $b / c$-hadrons}

\subsection{Beauty Hadron Masses}

The CDF Run II detector is in a unique position to greatly improve the measurement accuracy of many of the $b$-hadron masses. In particular, the measurement of the $B_{s}$ and beauty-baryon masses which are only accessible at the Tevatron. The calibration of the CDF II momentum scale is the key ingredient in precision mass measurements. The momentum scale is correctly determined by a complete accounting of the detector material for energy loss corrections, and a correct calibration of the detector magnetic field. The Run II momentum scale is calibrated using a large data sample of $538,000 \mathrm{~J} / \psi \rightarrow \mu \mu$ events. The variation 

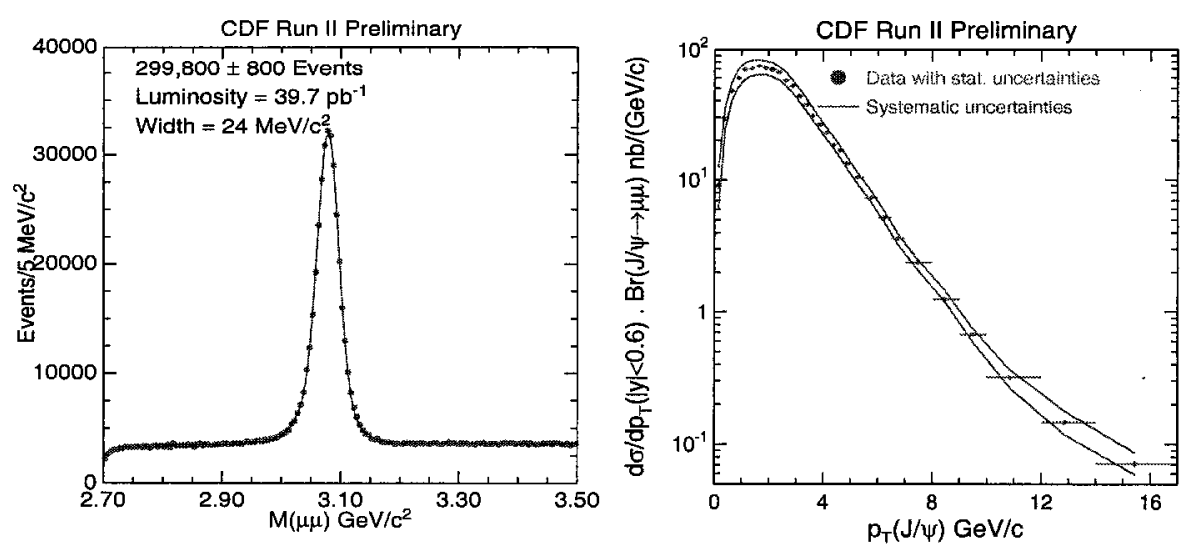

Fig. 5. The invariant mass distribution of triggered $J / \psi$ events reconstructed in $37 \mathrm{pb}^{-1}$ of Run-II data (left). The differential cross-section for $J / \psi$ with $|y|<0.6$ (right).

of the $J / \psi$ invariant mass as a function of transverse momentum is a very precise indicator of whether all material has been included properly. A small amount of passive material is added to the material description of the silicon vertex detector, until no variation in the $J / \psi$ invariant mass is seen as a function of transverse momentum, as shown in Figure 6. In addition the final calibration of the CDF magnetic field is performed by correcting the $J / \psi$ momenta until agreement is reached with the world average value (Figure 6) [5]. The calibra-

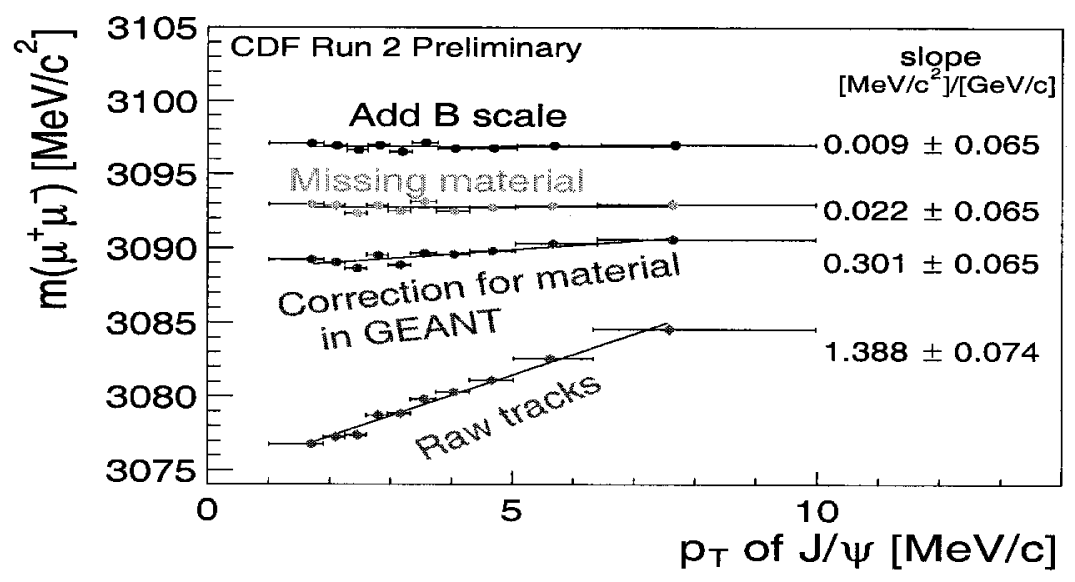

Fig. 6. The $J / \psi$ mass as a function of transverse momentum. Red points: No energy loss corrections, blue points: energy loss corrections using default material description, green points: energy loss correction using final calibration of silicon material, black points: invariant mass after magnetic field calibration applied. 
tions are cross-checked by applying the material and magnetic field corrections to other standard mass signatures: $K_{s}, D^{0, \pm}$, and $\Upsilon(1 S)$. These are found to be in excellent agreement with the PDG values. Figure 7 shows the invariant mass distributions of $B^{+} / B^{0} / B_{s} / \Lambda_{b}$, measured using the calibrated material and magnetic field, in fully reconstructed hadronic decays to final states containing a $J / \psi$. The invariant masses measured and the comparison to the PDG 2002
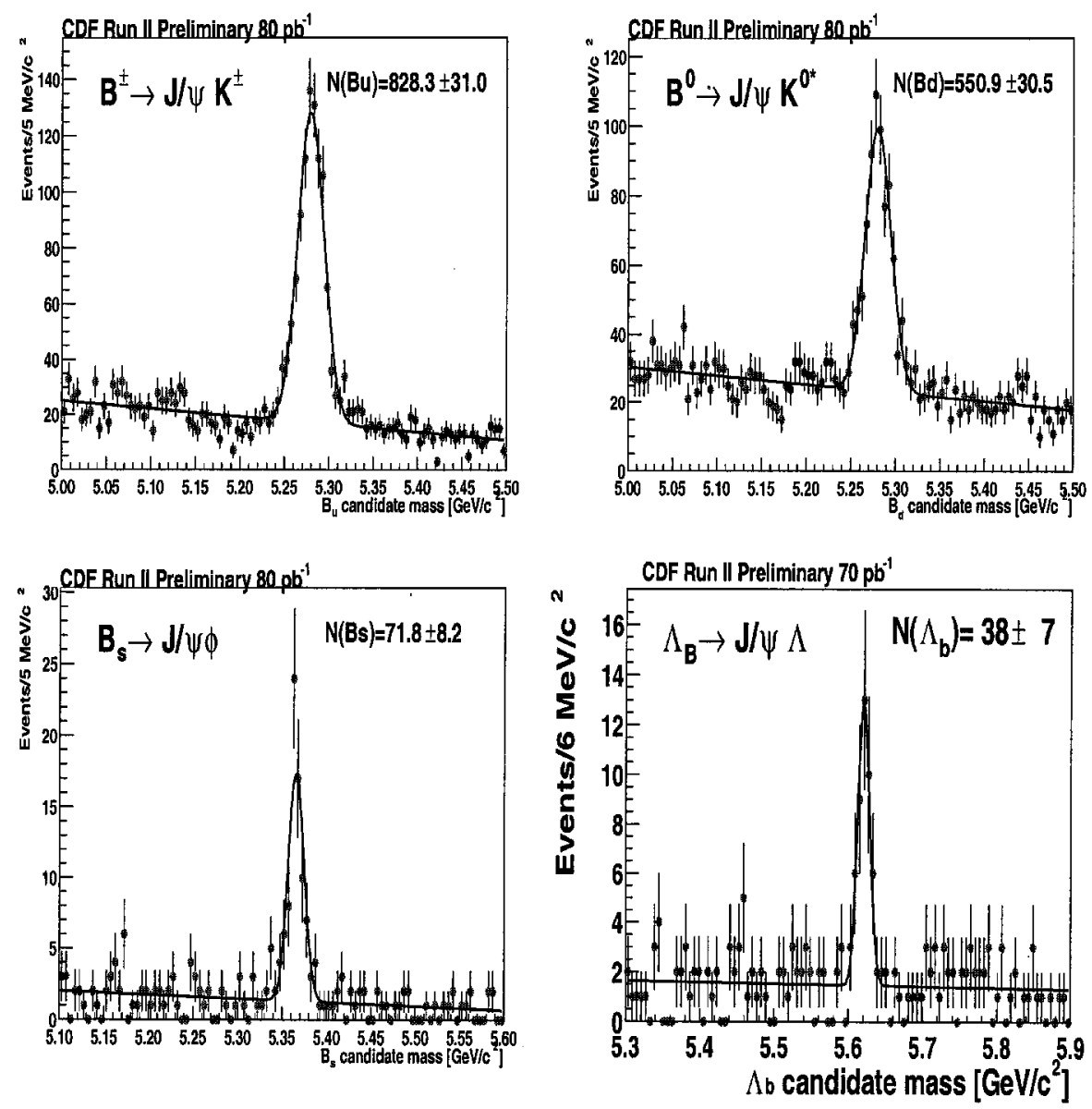

Fig. 7. $b$-hadron decay modes used to measure the $B^{+}, B^{0}, B_{s}^{0}$, and $A_{b}$ invariant mass in CDF Run II.

results [5] are listed in Table 1 . The CDF Run II measurements of $B_{s}$ and $A_{b}$ mass show marked improvement over the current world average results.

\section{2 $D_{s}^{+}-D^{+}$Mass Difference}

The measurement of $\Delta M\left(D_{s}^{+}-D^{+}\right)$is input to the overall PDG fit for all the charmed mesons. The values of the mass difference are predicted by Heavy Quark Effective Theory (HQET) [6] and Lattice QCD calculations [7]. The energy loss 


\begin{tabular}{ccc} 
Particle & Measured mass $\left(\mathrm{MeV} / \mathrm{c}^{2}\right)$ & PDG 2002 mass $\left(\mathrm{MeV} / \mathrm{c}^{2}\right)$ \\
\hline$B^{+}$ & $5279.32 \pm 0.68($ stat $) \pm 0.94($ syst $)$ & $5279.0 \pm 0.5$ \\
$B^{0}$ & $5280.30 \pm 0.92($ stat $) \pm 0.96($ syst $)$ & $5279.4 \pm 0.5$ \\
$B_{s}^{0}$ & $5365.50 \pm 1.29($ stat $) \pm 0.94($ syst $)$ & $5369.6 \pm 2.4$ \\
$\Lambda_{b}$ & $5620.4 \pm 1.6($ stat $) \pm 1.2($ syst $)$ & $5624 \pm 9$ \\
\hline
\end{tabular}

Table 1. $b$-hadron mass measurements at CDF II

and magnetic field calibrations described in the last section are applied to the measurement of the $D_{s}$ mass in the decay modes $D_{s}^{ \pm}, D^{ \pm} \rightarrow \phi \pi, \phi \rightarrow K K$. An unbinned $\log$-likelhood fit to the invariant mass of $\phi \pi$ is performed and the fit projection is shown in Figure 8.

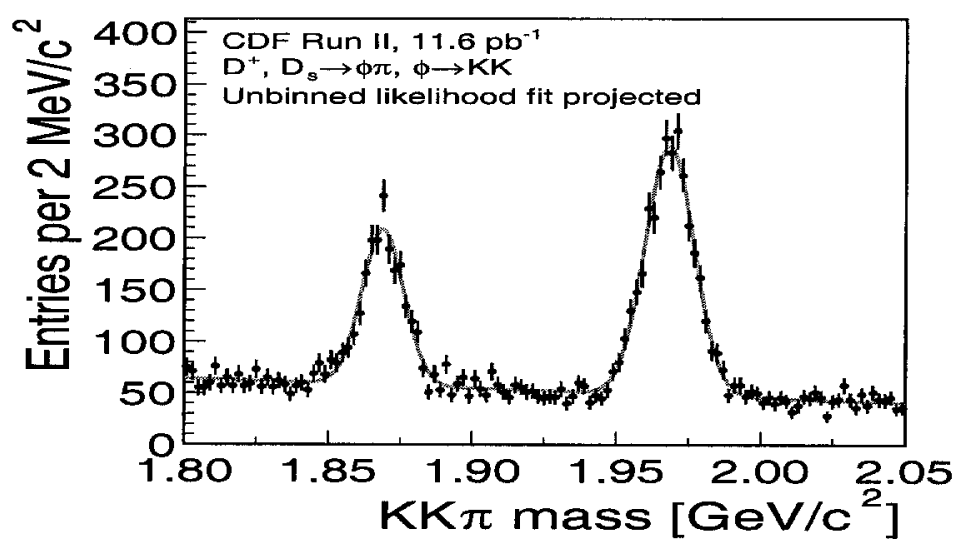

Fig. 8. The $D_{s}$ invariant mass distribution in data (points with error bars) and fit projection (histogram).

The mass difference measured is: $m\left(D_{s}^{ \pm}\right)-m\left(D^{ \pm}\right)=99.41 \pm 0.38($ stat $) \pm$ 0.21 (syst) $\mathrm{MeV} / \mathrm{c}^{2}$. This measurement is already an improvement over the current particle data group value of $m\left(D_{s}^{ \pm}\right)-m\left(D^{ \pm}\right)=99.2 \pm 0.5 \mathrm{MeV} / \mathrm{c}^{2} \quad[5]$.

\section{3 $\quad B_{s}$ lifetime using $B_{s} \rightarrow J / \psi \phi$}

The lifetime of the $B_{s}$ meson is the least well measured of the $B$ meson lifetimes making it difficult to compare experimental values of the lifetime ratios with theoretical prediction. In addition, theory predicts a lifetime difference between the two $B_{s} \mathrm{CP}$ eigenstates, which can be extracted by combining the lifetime measurement with an angular analysis. To this end, a new measurement has been performed of the $B_{s}$ lifetime at CDF in the decay mode $J / \psi \phi$, which is a mixed CP state. A total of $120 B_{s} \rightarrow J / \psi \phi$ events are reconstructed from $138 \mathrm{pb}^{-1}$ of data, and the lifetime is extracted from a maximum log likelihood fit to the proper decay length of $B_{s}$ candidates. The $B^{0}$ lifetime is measured 

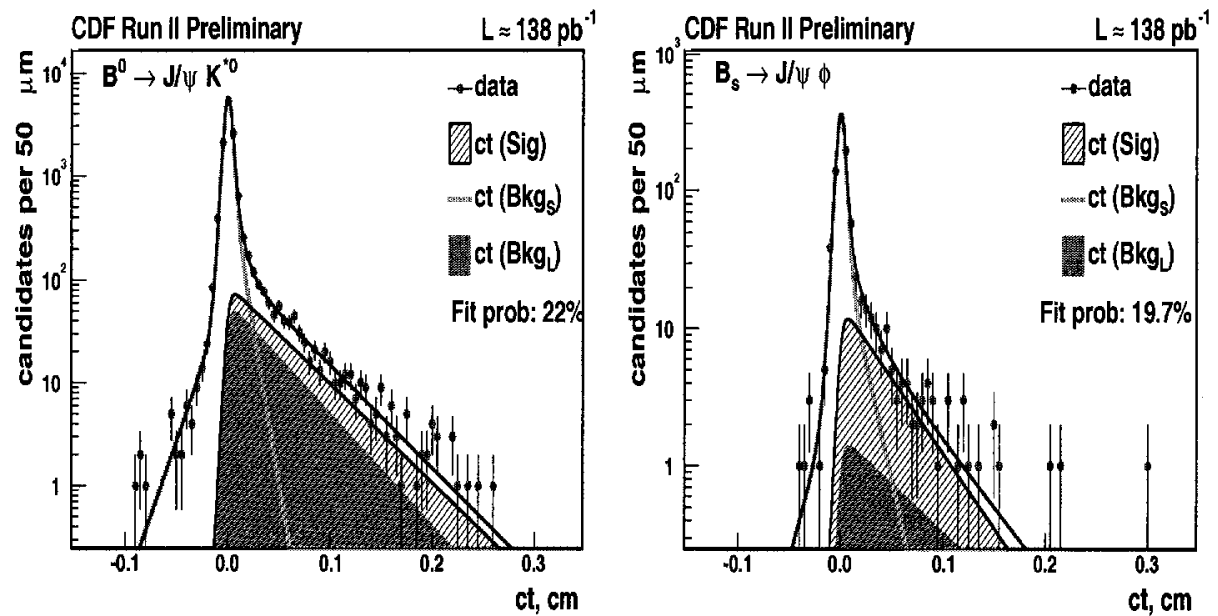

Fig. 9. Lifetime fit projection for $B^{0} \rightarrow J / \psi K^{*}$ (left) and $B_{s} \rightarrow J / \psi \phi$ (right).

in a similar fashion using a much larger sample of $800 B^{0} \rightarrow J / \psi K^{* 0}$ events. This sample is also used to estimate systematic uncertainties. The CDF Run II lifetimes of $B_{s}^{0}$ and $B^{0}$ are measured to be $\tau_{B_{s}}=1.33 \pm 0.14($ stat $) \pm 0.02$ (syst) $\mathrm{ps}$, and $\tau_{B^{0}}=1.51 \pm 0.06($ stat $) \pm 0.02$ (syst) ps respectively. The data and fit distributions are shown in Figure 9. An angular analysis is currently under-way to separate the two CP eigenstates using the larger data sample collected in Summer 2003.

\section{4 $\Lambda_{b}$ Lifetime, and Branching Fractions}

A combination of the increased luminosity of Run II and new triggers oriented towards $B$-physics has created new opportunities to study the heavy baryons and expand the knowledge of the structure of baryonic matter.

Prior to the start of Run II of the Tevatron, no measurement existed of the $\Lambda_{b}$ lifetime in a fully reconstructed mode. Lifetime measurements using partially semileptonic decay modes containing a $\Lambda_{c}$ and a lepton from LEP [8] and CDF Run I [9] were limited in accuracy and had feed-down from other heavy baryons. In CDF Run II, the $\Lambda_{b}$ lifetime in the fully reconstructed hadronic decay mode of $\Lambda_{b} \rightarrow J / \psi \Lambda$ has been measured. Using $46 \pm 9$ reconstructed events, the lifetime is measured to be $\tau_{\Lambda_{b}}=1.25 \pm 0.26$ (stat) \pm 0.1 (syst) ps. The current measurement is from $65 \mathrm{pb}^{-1}$ of Run II data. The statistical uncertainty on this measurement is expected to be greatly reduced when the full $225 \mathrm{pb}^{-1}$ of Run II data is analysed. The invariant $\Lambda_{b}$ mass and the corresponding lifetime fit results are shown in Figure 10 .

CDF Run II has successfully carried out new and improved measurements of the $\Lambda_{b}$ mass and lifetime using fully reconstructed decay modes containing $J / \psi$ obtained from the enhanced di-muon and level 1 track triggers. Heavy baryon decays to non-charmonium final states have also been reconstructed using data 

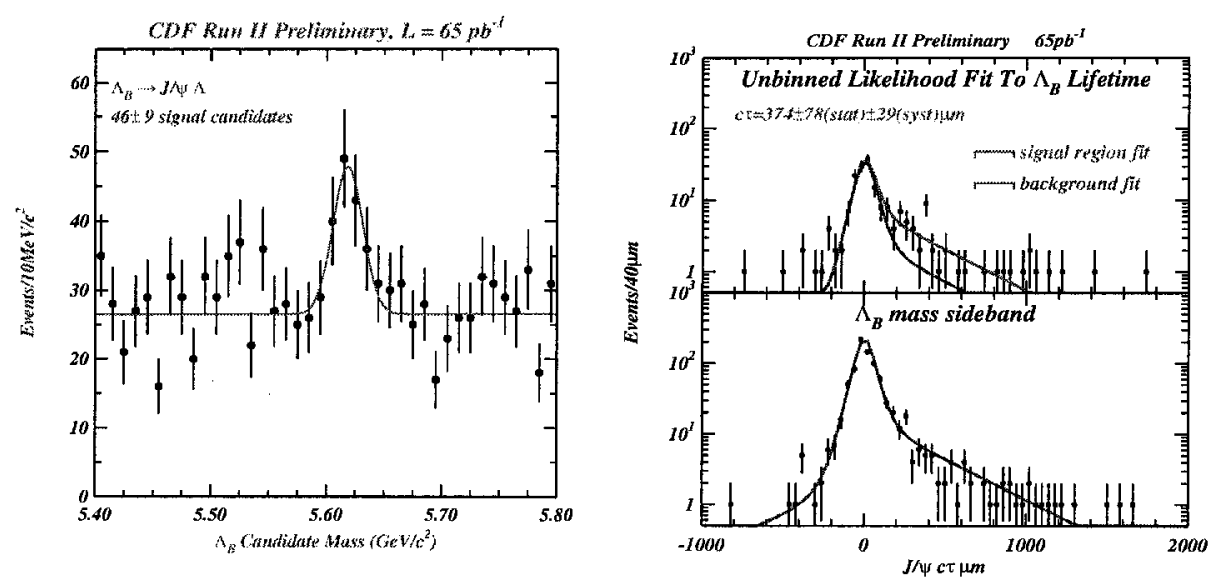

Fig. 10. Invariant mass distributions of $\Lambda_{b} \rightarrow J / \psi \Lambda$ events used to measure the $\Lambda_{b}$ lifetime (left). Projection of the unbinned log-likelihood fit to the $\Lambda_{b}$ proper decay length (right).

obtained from the silicon vertex trigger. A sample of $96 \Lambda_{b} \rightarrow \Lambda_{c}^{+} \pi^{-}$candidates has been reconstructed and is used to measure the branching fraction of $\Lambda_{b}$ in this decay mode. At CDF, measurements of ratios of branching ratios are more precise than absolute measurements, since most of the systematic errors cancel. It is then possible to rely on the $\mathrm{B}$ factories to provide precise measurements of higher-statistics branching ratios for use as denominators. Further, it is advantageous to package this branching ratio together with other poorly known quantities such as fragmentation fractions. The $\Lambda_{b} \rightarrow \Lambda_{c}^{+} \pi^{-}$measurement is normalized to the decay $B^{0} \rightarrow D^{-} \pi^{+}, D^{+} \rightarrow K \pi \pi$, which has the same number of particles in the final state. The decay $\Lambda_{b} \rightarrow \Lambda_{c}^{+} \pi^{-}$contains reflections and satellite contributions from other $B$ meson decays, and other baryons. All possible reflections are studied and the different components are put into a one-dimensional binned extended likelihood fit. The fit projections to the $\Lambda_{c}^{+} \pi^{-}$ distributions are shown in Figure 11. The 2002 PDG fragmentation ratio of $f_{\text {baryon }} / f_{d}=0.304 \pm 0.053$, where $f_{A_{b}}$ and $f_{d}$ are the fragmentation fractions to $\Lambda_{b}$ and $B_{d}$ respectively, is used to extract the ratio of branching fractions:

$$
\frac{B r\left(\Lambda_{b} \rightarrow \Lambda_{c}^{+} \pi^{-}\right)}{B r\left(B^{0} \rightarrow D^{-} \pi^{+}\right)}=2.2 \pm 0.4(\text { stat }) \pm 0.3(\text { syst }) \pm 0.7(B R+F R)
$$

This is the first measurement of the $\Lambda_{b}$ branching fraction in this mode.

\section{Rare Charm Decays}

\subsection{Cabbibo suppressed $D$ decays}

The study of the precise structure of the Standard Model has been guided by measurements of mixing and $\mathrm{CP}$ violation in the neutral $\mathrm{K}$ and $\mathrm{B}$ meson sectors. 


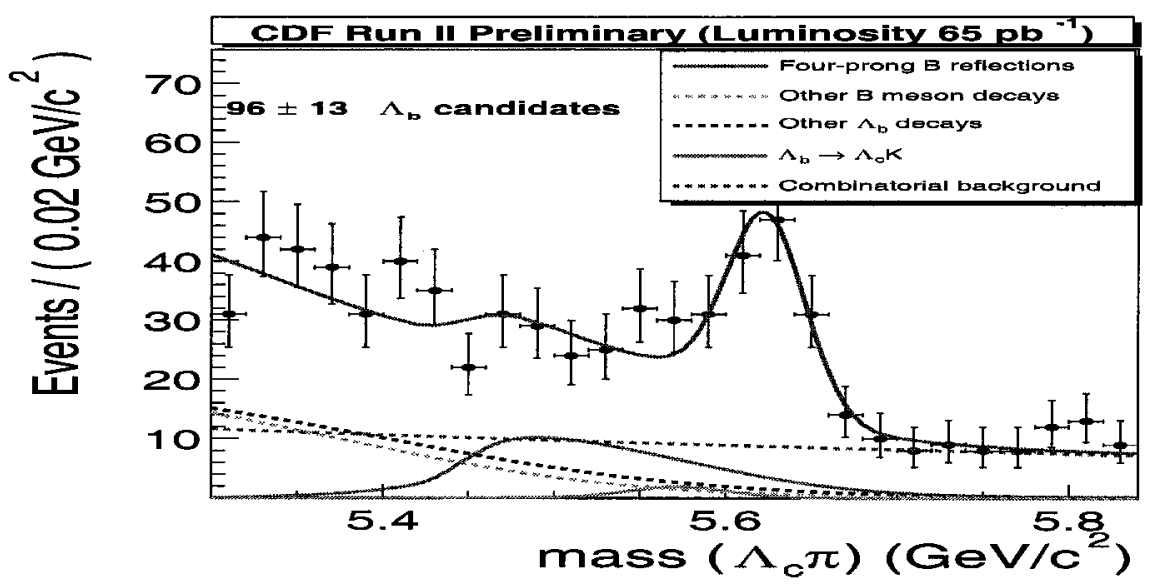

Fig. 11. Fit projections to the $\Lambda_{c}^{+} \pi^{-}$distributions including all reflections and satellite contributions from other $B$ meson decays.

The Standard Model predictions for the rate of mixing and CP violation in the charm sector are small, with the predictions in both cases ranging from 0.1$1.0 \%$ [10]. Observation of $\mathrm{CP}$ violation above the $1 \%$ level would be strong evidence for physics beyond the Standard Model. The SU(3) flavor symmetry predicts $\Gamma\left(D^{0} \rightarrow K^{+} K^{-}\right) / \Gamma\left(D^{0} \rightarrow \pi^{+} \pi^{-}\right)=1.0$, while the world average value is $2.88 \pm 0.15$. This deviation is most likely caused by large final state interactions. The observed $C P$ asymmetry in these decays is proportional to the direct $C P$ violation, which is predicted to be small in the SM, multiplied by the sine of the strong phase difference. $D^{0}$ candidates are combined with a soft pion, $\pi_{s}$, from $D^{* \pm} \rightarrow D^{0} \pi_{s}^{ \pm}$. The soft pion is used to tag flavor as well as reduce combinatorial background and remove the auto-reflection from $D^{0} \rightarrow K \pi$ decays. The distributions of the reconstructed decays are shown in Figure 12.

The ratios of branching fractions normalized to $D^{0} \rightarrow K \pi$ is measured to be $\frac{\Gamma\left(D^{0} \rightarrow K^{+} K^{-}\right)}{\Gamma\left(D^{0} \rightarrow K \pi\right)}=9.38 \pm 0.18($ stat $) \pm 0.10($ syst $) \%$ and $\frac{\Gamma\left(D^{0} \rightarrow \pi^{+} \pi^{-}\right)}{\Gamma\left(D^{0} \rightarrow K \pi\right)}=3.686 \pm$ 0.076 (stat) \pm 0.036 (syst) $\%$. These measurements compare favorably with the worlds current best measurements from the FOCUS [11] experiment of $\Gamma\left(D^{0} \rightarrow\right.$ $\left.K^{+} K^{-}\right) / \Gamma\left(D^{0} \rightarrow K \pi\right)=9.93 \pm 0.14 \pm 0.14 \%$ and $\Gamma\left(D^{0} \rightarrow \pi^{+} \pi^{-}\right) / \Gamma\left(D^{0} \rightarrow\right.$ $K \pi)=3.53 \pm 0.12 \pm 0.06 \%$. The intrinsic charge asymmetry of the CDF detector is corrected for by studying samples independent samples of unbiased tracks as a function of track transverse momentum. To check for any possible residual effects, the corrections are applied to the $D^{0} \rightarrow K \pi$ data sample where no CP asymmetry is expected. After correcting for detector effects, the decay asymmetries are found to be $\mathcal{A}_{c p}\left(D^{0} \rightarrow K K\right)=2.0 \pm 1.7$ (stat $) \pm 0.6($ syst $) \%$ and $\mathcal{A}_{c p}\left(D^{0} \rightarrow \pi \pi\right)=3.0 \pm 1.9$ (stat $) \pm 0.6($ syst $) \%$.

Given the systematic uncertainties currently achieved by these measurements, the addition of more data from CDF Run II should significantly improve the current measurement precision. 

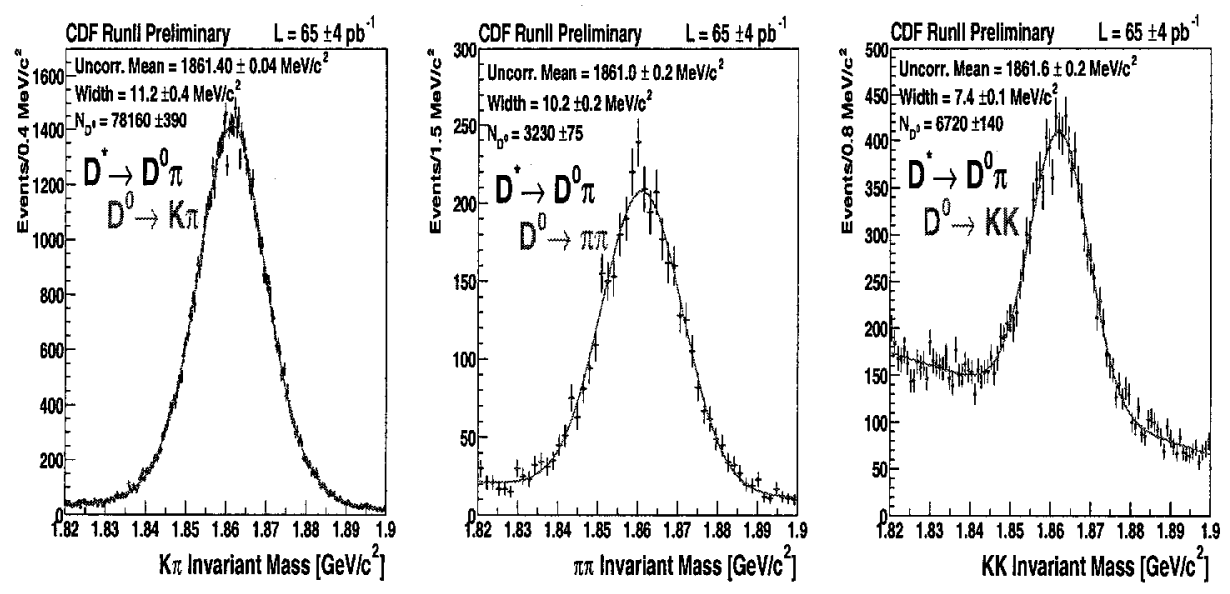

Fig. 12. The $D^{*}$ tagged cabbibo suppressed $D^{0}$ decay modes and the normalization $D^{0} \rightarrow K \pi$ decay mode.

\subsection{Rare FCNC $D^{0} \rightarrow \mu \mu$}

In the Standard Model, the FCNC decay $D^{0} \rightarrow \mu \mu$ is suppressed by the GIM mechanism, with a branching fraction estimated to be $\operatorname{Br}\left(D^{0} \rightarrow \mu \mu\right) \approx 3 \times 10^{-13}$ [12]. In contrast, some SUSY models with $R$-Parity violation predict branching fractions as large as $3.5 \times 10^{-6}$ [12]. The best published limit, at $90 \%$ C.L. is, $4.1 \times 10^{-6}$. Given the large number of charm mesons accessible at CDF Run II with the silicon vertex trigger, CDF Run II has the potential to take the lead in the search and improve over existing experiments. The SVT-triggered samples of $D^{0} \rightarrow K^{-} \pi^{+}$are used to understand acceptance, background and signal normalization. The results are normalized to the mode $D^{0} \rightarrow \pi^{+} \pi^{-}$such that most acceptance effects cancel and only the relative efficiency of $D^{0} \rightarrow \mu^{+} \mu^{-}$ to $\pi^{+} \pi^{-}$, obtained from a Monte Carlo simulation, is needed. The expected background is from $D^{0} \rightarrow \pi^{+} \pi^{-}$with both pions misidentified as muons and combinatorics. The $\pi / K$ mis-identification probability is determined from $D^{*}$ tagged $D^{0} \rightarrow K \pi$ events, where one or both tracks are tagged as muons and is shown in Figure 13. The expected combinatoric background contribution is obtained from a study of the mass sideband in $D^{*}$ tagged $D^{0} \rightarrow \pi^{+} \pi^{-}$events. The $D^{0} \rightarrow \pi^{+} \pi^{-}$normalization and mis-identification backgrounds are shown in Figure 14. The current background estimate is found to be 2 events and is dominated by combinatoric backgrounds. The number of events found in the search window after muon identification is required on both tracks is zero as shown in Figure 14, therefore the current CDF limit obtained from $63 \mathrm{pb}^{-1}$ of data is $\operatorname{Br}\left(D^{0} \rightarrow \mu^{+} \mu^{-}\right) \leq 2.4 \times 10^{-6} @ 90 \%$ C.L. A better understanding of the sources of combinatoric background could potentially increase the sensitivity of the current analysis to $2 \times 10^{-7}$. 

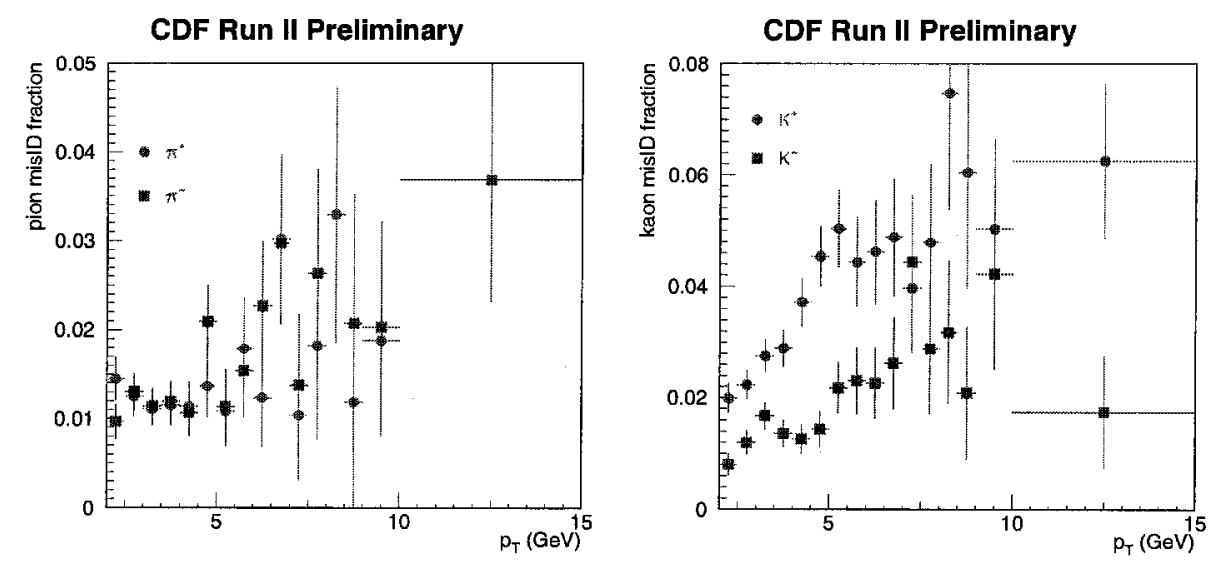

Fig. 13. Using $D^{*}$ tagged $D^{0} \rightarrow K \pi$ decays, the probability that a pion (left) or kaon (right) is misidentified as a muon is measured. The transverse momentum dependence is shown.
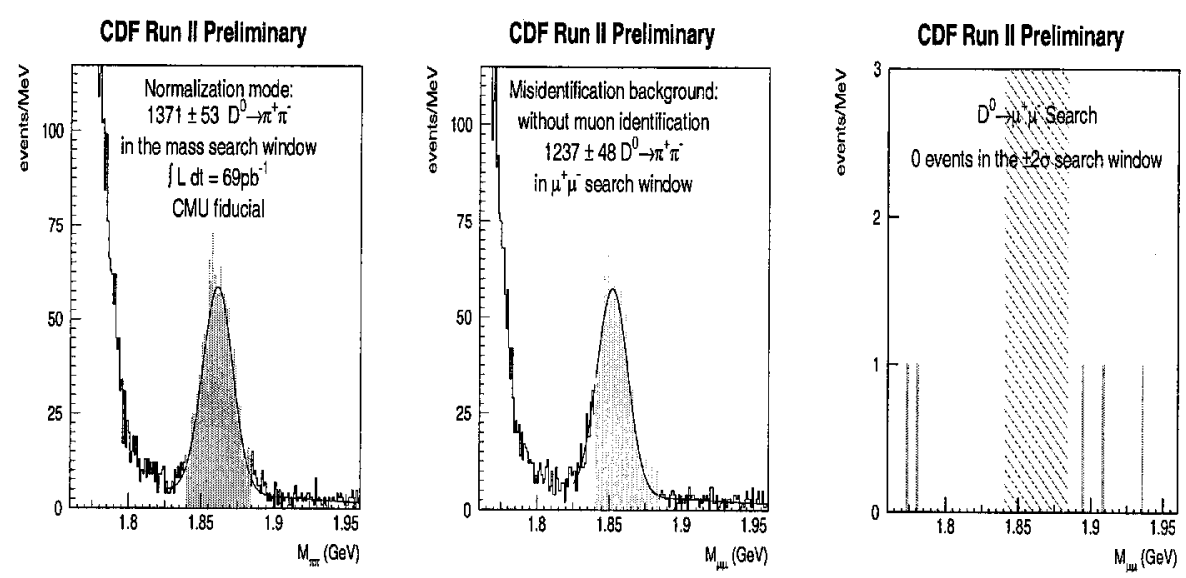

Fig. 14. The $D^{*}$ tagged $D^{0} \rightarrow \pi^{+} \pi^{-}$used for normalization (left). The $D^{0} \rightarrow \pi^{+} \pi^{-}$mis-identification background without muon identification required (center) and the $\mu \mu$ invariant mass after both tracks are required to be tagged as muons (right). 

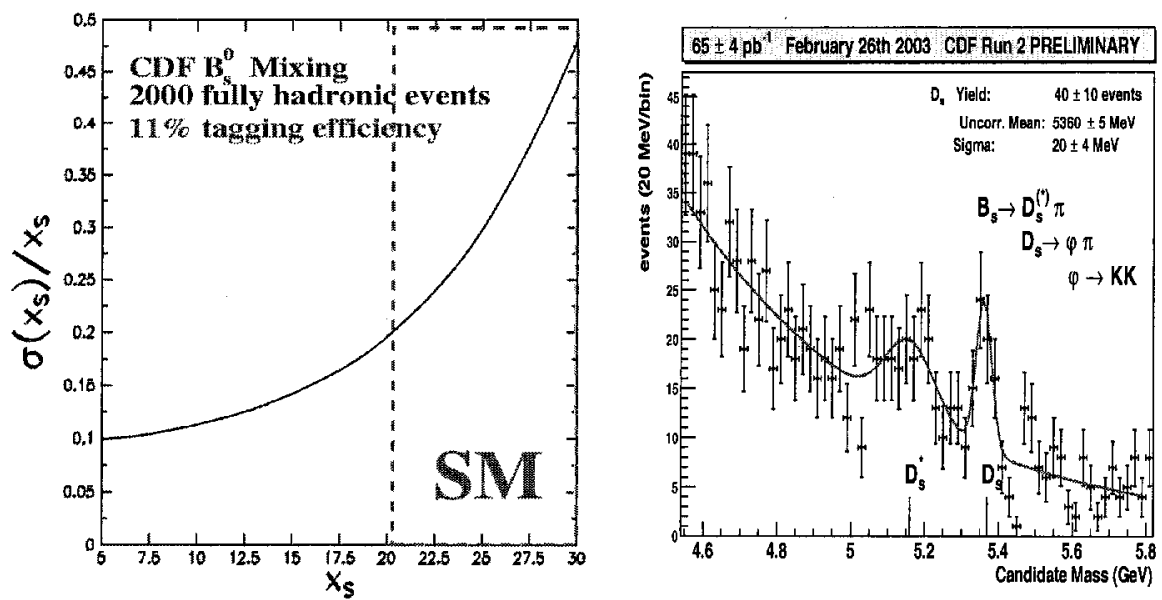

Fig. 15. $B_{s}$ mixing reach using 2000 fully reconstructed hadronic $B_{s}$ decays (left). $B_{s} \rightarrow D_{s} \pi$ signals reconstructed in the first $65 \mathrm{pb}^{-1}$ of CDF Run II data (right).

\section{CKM Physics Prospects in CDF Run II}

\section{$5.1 \quad B_{s}$ mixing}

In Figure 15, the $B_{s}$ mixing reach from 2000 fully reconstructed hadronic decays, assuming an $11 \%$ tagging efficiency is shown. The first $B_{s} \rightarrow D_{s} \pi$ signals reconstructed using the non-optimized silicon tracker performance shown in Figure 1 is shown in Figure 15. Using the current improved performance of the track triggers and the same signal to background of $2 / 1,1600$ hadronic events per $\mathrm{fb}^{-1}$ are expected, extrapolating from the already reconstructed $B_{s} \rightarrow D_{s} \pi$ signal. With modest improvements in triggers, more hadronic modes reconstructed, and including kaon tagging using the new time-of-flight system in CDF Run II, the 2000 events of fully hadronic $B_{s}$ decays are achievable in $1 \mathrm{fb}^{-1}$ - this gives a 5 $\sigma$ sensitivity for $\Delta\left(m_{s}\right)=18 \mathrm{ps}^{-1}$ with $1.7 \mathrm{fb}^{-1}$ of data and a $5 \sigma$ sensitivity for $\Delta\left(m_{s}\right)=24 \mathrm{ps}^{-1}$ with $3.2 \mathrm{fb}^{-1}$.

\subsection{Direct CP Violation in $b \rightarrow h h$}

CDF Run II has collected several hundred charmless two track decays of $B$ mesons in the decay mode $b \rightarrow h h$ events, where $h$ could be $\pi / K / p$ and $b$ could be a $B^{+} / B^{0} / B_{s} / \Lambda_{b}$. The invariant mass distribution of $b \rightarrow h h$ candidates from $65 \mathrm{pb}^{-1}$ using a pion hypothesis for both tracks is shown in Figure 16. The goal is to measure the CP decay asymmetries in the decay modes $\mathcal{A}_{c p}\left(B_{d} \rightarrow \pi^{+} \pi^{-} / K^{+} \pi^{-}, B_{s} \rightarrow K K / K^{-} \pi^{+}, \Lambda_{b}^{0} \rightarrow p^{+} \pi^{-} / p^{+} K^{-}\right)$. Although the CP asymmetries in the charmless 2-body $B_{d}$ decay modes have been measured at the $B$-factories, the asymmetries in the modes $B_{s}$ and $A_{b}$ are currently only accessible at the Tevatron. The first step in measuring the CP asymmetries is disentangling the different components that contribute $b \rightarrow h h$. Differences in 


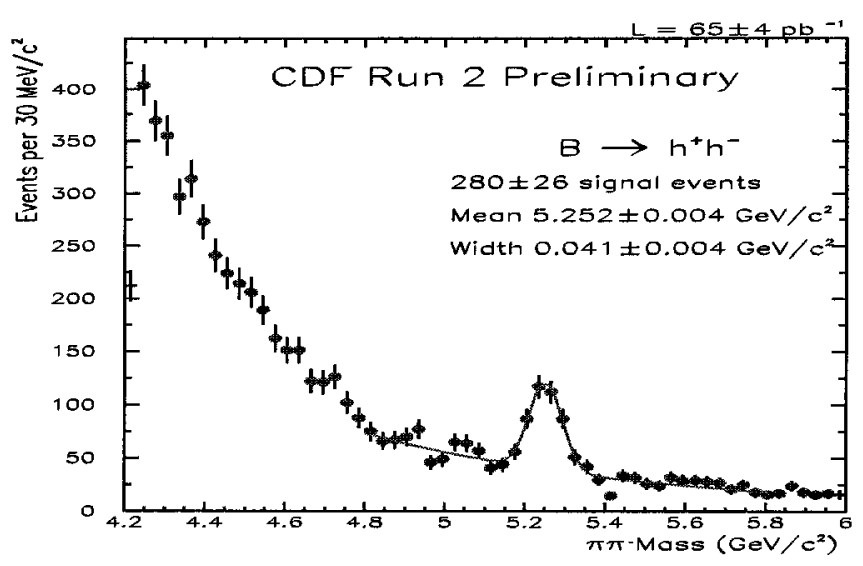

Fig. 16. $b \rightarrow h h$ invariant mass distributions using a pion hypothesis

$\pi-K$ kinematics are used to separate $K \pi / \pi K$ from $\pi \pi / K K$ by exploiting the relationship between the invariant mass and the quantity $\alpha=\left(1-p_{1} / p_{2}\right) \cdot q_{1}$, where $p_{1} / p_{2}$ is the ratio of the minimum to the maximum momentum of the two decay hadrons and $q_{1}$ is the charge of the lowest momentum hadron. The distribution of $\alpha$ versus $M(\pi \pi)$ from a Monte Carlo simulation is shown in Figure 17. In addition, $\pi / K$ separation using $\mathrm{dE} / \mathrm{dx}$ particle id is used to separates $\pi \pi$ decays from $K K$, and $\pi K$ from $K \pi$. An unbinned $\log$-likelihood fit to the kinematic variables $\alpha, M(\pi \pi)$, and the $\pi / K \mathrm{dE} / \mathrm{dx}$ probabilities are used to separate the different $h h$ components. The fractions of $B^{0} \rightarrow K \pi, B^{0} \rightarrow \pi \pi, B_{s} \rightarrow K K, B_{s} \rightarrow K \pi$ are $53 \pm 6 \%, 14 \pm 5 \%, 32 \pm 6 \%$, and $1 \pm 4 \%$ respectively. The results are used to extract the decay asymmetry in the mode $B^{0} \rightarrow K \pi$ :

$$
\mathcal{A}_{C P}=\frac{\bar{B}_{d}^{0} \rightarrow K^{-} \pi^{+}-B_{d}^{0} \rightarrow K^{+} \pi^{-}}{\bar{B}_{d}^{0} \rightarrow K^{-} \pi^{+}+B_{d}^{0} \rightarrow K^{+} \pi^{-}}=0.02 \pm 0.15 \text { (stat) } \pm 0.017 \text { (syst) }
$$

and the relative branching fraction of $B_{s} \rightarrow K K$ :

$$
\frac{B r\left(B_{s} \rightarrow K^{ \pm} K^{\mp}\right)}{B r\left(B_{d} \rightarrow K^{ \pm} \pi^{\mp}\right)}=2.71 \pm 0.73(\text { stat }) \pm 0.35\left(f_{s} / f_{d}\right) \pm 0.81(\text { syst })
$$

using the world average measurement of the fragmentation fraction $f_{s} / f_{d}=$ $0.27 \pm 0.04$.

\section{Conclusion}

CDF Run II has produced new measurements of the charm and beauty crosssections, lifetimes and branching fractions using the data sample collected during the first year of running. These measurements exploit the high yields of triggered displaced charm mesons and $J / \psi$ from the secondary vertex track trigger and upgraded dimuon triggers respectively. More than 1 million charm and $J / \psi$ signals 

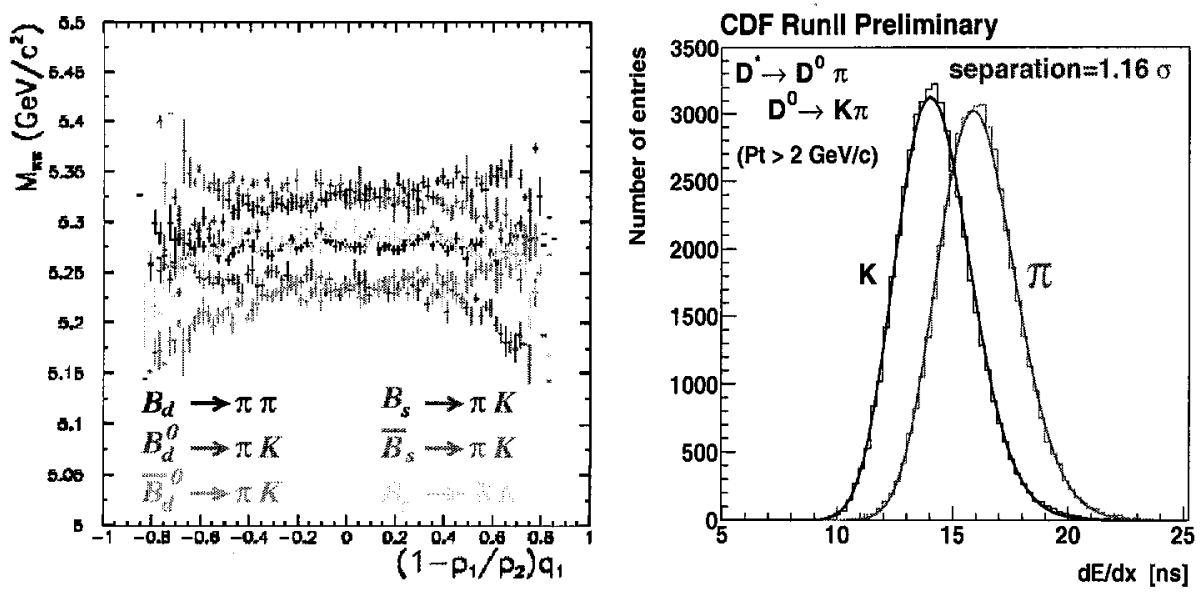

Fig. 17. The $h h$ invariant mass using a pion hypothesis for various $b \rightarrow h h$ decays versus the quantity $\alpha=\left(1-p_{1} / p_{2}\right) \cdot q_{1}$ (left). On the right is the CDF $K-\pi \mathrm{dE} / \mathrm{dx}$ separation.

have been reconstructed. The upgraded detector and triggers have enabled CDF Run II to significantly contribute new knowledge of the heavier beauty hadrons which are currently accessible only at the Fermilab Tevatron. New measurements of the $B_{s}$ and $A_{b}$ masses, lifetimes and branching fractions have improved or superseded the current worlds best measurements. The study of rare charm decays has been established as a tool to probe physics beyond the Standard Model, and a new best limit on the rare FCNC decay of $D^{0} \rightarrow \mu \mu$ has been measured. In addition the decay signals $B_{s} \rightarrow D_{s} \pi$ and the two body charmless decays of $B^{0}$ and $B_{s}$ have been established. These decay channels are important milestones towards the measurement of $B_{s}$ mixing and CP violating decay asymmetries.

\section{References}

1. The CDF II Detector Technical Design Report FERMILAB-Pub-96/390-E.

2. W. Ashmanskas et al. Nucl. Instrum. Meth. A501,201-206, (2003).

3. Mateo Cacciaro, Mario Greco, and Paolo Nason. JHEP, 05:007, 1998

4. Adam K. Leibovich, Nucl. Phys. Proc. Suppl. 93182 (2001).

5. K. Hagiwara et. al., Physical Review D 66, 010001 (2002).

6. S. Godfrey and N. Isgure, Phys. Rev. D94, 189 (1985).

7. R. Lewis and R. M. Woloshyn, Phys. Rev. D 58, 074506 (1998).

8. LEP B lifetimes working group results at http://lepbosc.web.cern.ch/LEPBOSC/lifetimes/lepblife_page2.html

9. F. Abe et al. Phys.Rev.Lett. 77, 1439 (1996).

10. H.N. Nelson, hep-ex/9908021; A. A. Petrov, hep-ph/0009160; I.I. Bigi hepex/0104008; A. F. Falk et al. hep-ph/0110317

11. FOCUS Collaboration (J.M. Link et al.) Phys. Lett. B555:167-173, (2003).

12. G. Burdman, E. Golowich, J. Hewett, S. Pakvasa, hep-ph/0112235 v2 (March 2002). 